\title{
Towards Designing an Integrated Earth Observation System for the Provision of Solar Energy Resource and Assessment
}

\author{
P. W. Stackhouse, Jr. \\ NASA Langley Research Center \\ Hampton, VA, 23681 \\ Email: paul.w.stackhouse@nasa.gov
}

D. Renné

National Renewable Energy Laboratory

1617 Cole Boulevard

Golden, CO 80402

H.-G. Beyer

Institute of Electical Engineering

University of Applies Sciences Magdeburg-Stendal (FH)

Breitscheidstrasse 2

D-39114 Magdeburg, Germany

\author{
L. Wald \\ Centre Energetique et Procedes
}

Ecole des Mines de Paris / Armines / CNRS (FRE 2861)

BP 207, F-06904 Sophia Antipolis cedex

\author{
R. Meyer \\ M. Schroedter-Homscheidt \\ DLR - Oberpfaffenhofen \\ Institut für Physik der Atmosphäre \\ D-82234 Wessling, Germany
}

\author{
M. Š́ri \\ European Commission \\ DG Joint Research Centre \\ Ispra, Italy
}

\begin{abstract}
The GEOSS strategic plan specifically targets the area of improved energy resource management due to the importance of these to the economic and social viability of every nation of the world. With the world's increasing demand for energy resources, the need for new alternative energy resources grows. This paper overviews a new initiative within the International Energy Agency that addresses needs to better manage and develop solar energy resources worldwide. The goal is to provide the solar energy industry, the electricity sector, governments, and renewable energy organizations and institutions with the most suitable and accurate information of the solar radiation resources at the Earth's surface in easily-accessible formats and understandable quality metrics. The scope of solar resource assessment information includes historic data sets and currently derived data products using satellite imagery and other means. Thus, this new task will address the needs of the solar energy sector while at the same time will serve as a model that satisfies GEOSS objectives and goals.
\end{abstract}

Keywords-component; energy; solar energy; renewable energy; GEOSS

Funding for the main author's work is provided through the NASA Applied Science Program Energy Management Theme; Co-author funding is provided through respective governments under the IEA agreement.

\section{INTRODUCTION}

Worldwide energy demand is growing rapidly as many previously underdeveloped nations continue industrialization. Coupled with political instability, natural disasters and potential power grid system breakdowns, the sensitivity and strain on world energy resources is high. With the worldwide growth in energy demand, there comes an increasing need to develop and use alternative energy sources. For this reason the Global Earth Observation Summit and the resulting plan to develop a Global Earth Observation System of Systems (GEOSS) program specifically targets the theme of energy resource management [1]. One of these alternate sources is solar energy. For instance the photovoltaic industry (PV) experiences solid growth at $30+\%$ per year is now approaching $\$ 10$ billion business worldwide. Solar electric technologies and energy from other renewable forms (i.e., wind, geothermal) are prospective grid supporting technologies, where the influx of solar generated electricity on worldwide power grids could be highly beneficial, depending on the characteristics of the solar resource. Thus, the characteristics of the solar resource along with it's 
variability and potential long-term changes will become increasingly important to the managing of energy resources, a stated GEOSS theme. Since the determination of the solar resource depends upon factors involving the sun, the atmosphere and earth's surface, the design of integrated earth observations systems using satellite information and modeling analysis to provide this information from an historic, near-real time and eventually a forecasted quantity will become an achievable goal.

In this paper, a brief overview of the state GOESS strategic plans for energy resource management is reviewed. Then, as new task under the International Energy Agency is described that is aimed at meeting the needs of solar energy sector and will also satisfy the goals and objectives of GOESS for the area of solar energy.

\section{GEOSS AND ENERGY RESOURCE MANAGEMENT}

In February 2005, the Group on Earth Observations, published a 10-year reference document [1] that resulted from the Earth Observation Summit, the first of which was held in 2003.

\section{A. GEOSS Purpose and Goals}

In this document [1], over 60 nations and the European Union agreed on the following:

- Improve coordination of strategies and systems for observations of the Earth and identify measures to minimize data gaps, with a view to moving toward a comprehensive, coordinated, and sustained Earth observation system or systems.

- Coordinate an effort to involve and assist developing countries in improving and sustaining their contributions to observing systems, as well as their access to and effective utilization of observations, data and products, and the related technologies by addressing capacity-building needs related to Earth observations.

- Exchange observations recorded from in situ, aircraft, and satellite networks, dedicated to the purposes of this Declaration, in a full and open manner with minimum time delay and minimum cost.

To reach these goals, a Global Earth Observation Systems of Systems was envisioned whereby the cooperating nations of the world would coordinate to prepare and dissemination important environmental information to meet the needs of societies around the world. GEOSS is aimed to provide information in 9 benefit theme areas. One of which is energy.

\section{B. Energy theme goals and targets}

The GEOSS goals and targets related to Energy Resource solar management are divided into 2, 6, and 10 year goals. The emphasis of the 2 year goals are focused upon facilitating the "exchange and use of existing data/products and forecast information" by raising awareness, facilitating access and training for the use thereof in decision support tools. Another of the goals is to produce a 5-10 year plan to develop future capabilities toward developing, disseminating and using those products. The 6-year goals involve the evaluation of the progress made against the original plan and to "continue the use of improved weather and climate products for the development of new energy tailored products and services". Lastly, the 10-year targets include the "implementation of appropriate operational observing systems" to support continued energy operations. This includes developing planned advances to this system. The aims of the new and the future improved operational system are to "facilitate the exchange of data and products for efficient energy management" and "facilitate the development of renewable energy systems taking advantage of GEOSS products."

\section{SOlar ENERgy Resource AsSESSMENT}

Current resource assessment maps are comprised of satellite based maps from different sources and methodologies. Some maps are generated by the interpolation of surface site measurements or from observations of sky cover. Some of these measurements do not have continuous records or verifiable quality control. The resulting maps tend to be detailed for limited regions and many are hard to obtain, especially for developing nations. Owing to the growing reliance on solar and other renewable energy technologies worldwide and the current state of the solar resource information, a task was formed under the International Energy Agency (IEA) entitled "Solar Resource Knowledge Management"[2] with the following three objectives:

- Provide standardization and benchmarking of international solar resource data sets,

- Provide improved data reliability, availability and accessibility in formats that address specific user needs, and

- Develop and improve methods so that resource products, including solar resource forecasts, achieve greater accuracy with higher temporal and spatial resolution

These objectives are complimentary to the goals of GEOSS and as such this project will provide a prototype example for the development of an integrated observing system that can be developed and optimized to provide the necessary solar resource information. Many other environmental variables required for the production of and optimization of energy resource use are potentially available within this framework or by adding appropriate components. 
Task 36 "Solar Resource Knowledge Management" (SRKM) was developed as a concept paper at a satellitebased solar resource assessment workshop in Switzerland in March 2003, and was approved at the $57^{\text {th }}$ meeting of the SHC Executive Committee (ExCo) in Espinho, Portugal, 1517 June 2005. Six countries (Canada, France, Germany, Spain, Switzerland, and the United States) and the European Union are officially participating in the task.

\section{A. SRKM Approach}

The international institutions participating in Task 36 are addressing these objectives through a co-coordinated work plan that encompasses three subtasks [2]:

1) Subtask A: Standardize and Benchmark Solar Resource Products to Ensure Worldwide Intercomparability and Acceptance (Subtask Leader: Dr. Hans-Georg Beyer)

The objectives of this Subtask are to provide:

- Coherence and benchmarking of models producing solar irradiance values from satellite data;
- Accessibility and coherence of ancillary model input data such as atmospheric conditions and land surface parameters;

- Sensitivity analyses;

- Ground truth validations with high quality data;

- Definition of validation protocols and measures of end-product confidence;

- Cross-satellite platform and cross-model comparisons

2) Subtask B: Develop Common Structure for Archiving and Accessing Data Products (Subtask Leader: Dr. Lucien Wald)

The objectives of this Subtask are to develop and produce deliverables designed to provide:

- Development of worldwide networking between distributed data centers resulting in a global

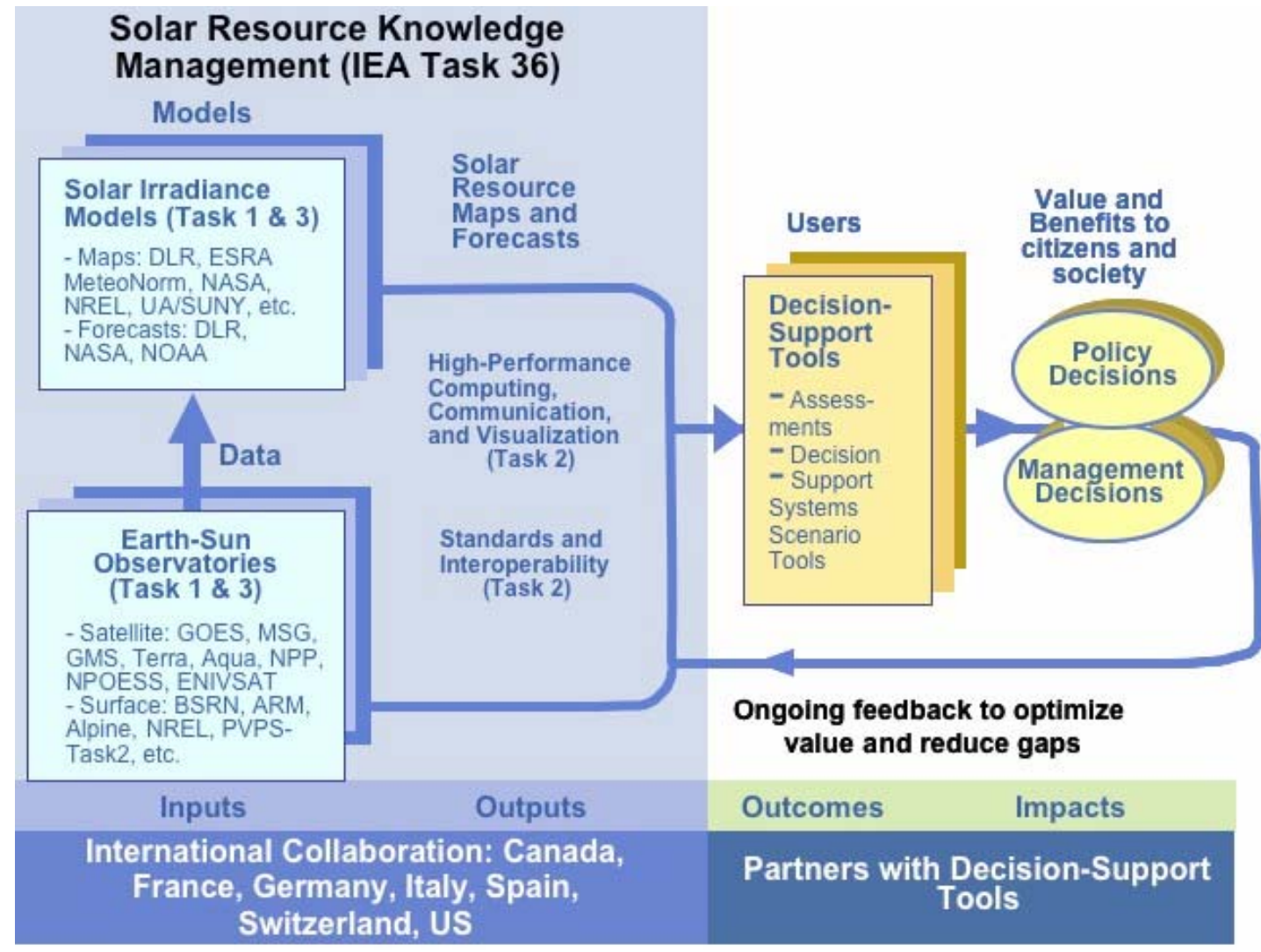

Figure 1: A integrated system view of the Solar Resource Knowledge Management task under the International Energy Agency. The chief duty of the activities under this task are shown on the left hand side of the plot. The effect on the users is located on the right. Observations of the Earth and Sun are needed to compute the available solar resource on a global basis. Acronyms for observations: GOES - Geosynchronous Orbiting Environmental Satellite (NOAA-US), MSG - MeteoSat $2^{\text {nd }}$ generation (EU), GMS - Global Meteorological Satellite (Japan), Terra and Aqua are polar orbiting NASA satellites, ENVISAT is a European Space Agency satellite. Surface observations include high quality solar radiation measurements made for the networks listed. 
coverage for high-quality solar resource data;

- Development of information and data exchange protocols;

- Reliable and fast end-user access;

- Preparation of data documentation for specific enduser applications.

3) Subtask C: Improve Techniques for Solar Resource Characterization and Forecasts (Subtask Leader: Dr. Richard Meyer)

The objective of this Subtask is to develop and produce deliverables designed to provide:

- Short-term (hours) to medium-term (days) solar resource forecasting;

- Analysis of long-term variability of solar resources;

- Improved exploitation of existing satellite resources (e.g. very high spatial resolution for plant micro siting);

- Adaptation of resource assessment techniques to the capabilities of new generations of satellites;

- Development of new methods to provide improved products like spectrally- or angular-resolved information.

The project and it's subtasks are shown in schematic in Figure 1. This figure shows the relationships between the inputs, outputs, the various tasks and the users.

\section{B. SRKM Status}

Since the official start of the 5-year Task on July 1, 2005, the first Task Experts Meeting, which was held in DLROberpfaffenhofen on 16-18 November. Approximately 22 Task Participants attended the Experts Meeting, including representatives from all participating countries (and the EU) except Canada. In addition, there was guest representation from Brazil, PVPS Task 2, and the European Space Agency. Substantive planning for each of the three major subtasks was accomplished at this meeting. A Second Experts Meeting was scheduled to be held in Denver on July 6-7, 2006 in advance of the Solar 2006 Conference. These meetings will determine the specific activities that the participating nations will strive to complete in collaboration.

SRKM has strong links with industry. Two small companies are directly participating in the task. The audience for the results of Task 36 includes the technical laboratories, research institutions, and universities involved in developing solar resource data products. More importantly, data users, such as energy planners, solar project developers, architects, engineers, energy consultants, product manufacturers, and building and system owners and managers, and utility organizations, are the ultimate beneficiaries of the research, and will be informed through targeted reports, presentations, web sites, handbooks and journal articles.

\section{Outreach to the GEOSS}

In December 2005 a SRKM letter of introduction was sent to the Secretariat of the Global Earth Observation System of Systems (GEOSS) headquarters office at the World Meteorological Organization in Geneva. The letter suggests possible ways that the Task supports GEOSS goals and explores mechanisms for participation in joint IEA/GEOSS activities. Members of SRKM are actively engaged in the development of GEOSS activities, and it is expected that some of the SRKM activities will contribute directly GEOSS objectives for energy.

\section{SUMmary AND CONCLUSION}

The GEOSS implementation plan calls for the development of the capacity to produce, disseminate and use earth, weather and climate data products to meet societal needs in 9 themed areas. One of those themes involving the managing of Earth resources directly calls for data products and services in energy and renewable technologies to contribute to the development of more energy efficient systems and the implementation of renewable energy technologies.

Parallel to this effort, the solar energy resource assessment community was working to improve both surface and satellite based solar irradiance data products, to evaluate and benchmark those products and to disseminate them to the users of solar energy systems. The collaboration of this community resulted in the development of the Solar Resource Knowledge Management task that is now officially sanctioned by the International Energy Agency. This task focuses on the development, validation, and access to solar resource information derived from surface-based and satellite-based platforms. It investigates benchmarking and data quality assessment procedures for data products and validation data sets, examines means by which the data can be made easily available to users through various web-based hosting schemes, and conducts studies on improving the input data sets and algorithms from which satellite-derived products are produced, including the investigation of short term forecasting and past and future climatic variability of the solar resource. Thus, this task directly parallels the goals of GEOSS and should represent a contribution of the IEA to GEOSS.

\section{REFERENCES}

[1] Global Earth Observation System of Systems (GEOSS): 10 Year Implementation Plan Reference Document, Bruce Battrick, Ed., ESA Publications Division, Noordwijk, Netherlands, GEO 1000R/ESA SP-1284, , 210 pp., February 2005

[2] Renné, D., H._G. Beyer, L. Wald, R. Meyer, "Solar Resource Knowledge Management: A new task of the International Energy Agency", (in press Proc. of American Solar Energy Society) 